\title{
Translational Research Grants Scheme (TRGS): a new approach to strengthening health system research capacity
} \author{
Antonio Pennab \\ a Centre for Epidemiology and Evidence, NSW Ministry of Health, Sydney, Australia \\ b Office for Health and Medical Research, NSW Ministry of Health, Sydney, Australia \\ c Sax Institute, Sydney, NSW, Australia \\ d Corresponding author: Robin.Auld@health.nsw.gov.au
}

Robin Aulda,d, Tina Loppacher ${ }^{b}$, Shiho Rose ${ }^{c}$, Andrew J Milat ${ }^{a}$ and

\section{Article history}

Publication date: September 2018 Citation: Auld R, Loppacher T, Rose S, Milat AJ, Penna A. Translational Research Grants Scheme (TRGS): a new approach to strengthening health system research capacity. Public Health Res Pract. 2018;28(3):e2831818. https://doi. org/10.17061/phrp2831818

\section{Key points}

- The Translational Research Grants Scheme in New South Wales (NSW), Australia, empowers NSW Health organisations to drive research according to local and state priorities, and to implement interventions and changes in their service

- This review describes the scheme and the key themes to come out of an initial implementation review, including the importance of Chief Executives' commitment to implementation of research findings

\section{Abstract}

Background: Bridging the 'gap' between research evidence and the complexities of policy and practice is central to health improvement. The Translational Research Grants Scheme (TRGS) in New South Wales (NSW), Australia, is a funding scheme aimed at reducing the time between research generation and translation to policy and practice. The TRGS is also an important part of NSW Health's efforts to harness and strengthen research capacity to improve health service delivery.

Methods: A document review and interviews $(n=12)$ with key stakeholders were undertaken following the first round of TRGS funding in November 2016. The communications from continuing quality improvement processes over the three funding rounds have provided further insight.

Results and discussion: A total of 53 projects have been funded under the scheme, with recipients across many NSW Health organisations. NSW Health has committed more than $\$ 24$ million to date. Round one of the TRGS was received well by the policy makers, Local Health Districts and research stakeholders interviewed. Of particular note were: the requirement for Chief Executives to demonstrate strong support for the implementation of findings; requirements to partner with state-wide policy leads and clinical networks; and capacity-building outcomes of the scheme. The ongoing quality improvement processes indicate that the program continues to be well received, with improvements to partnership arrangements, and an acknowledgement of the challenge that arises because the scheme, by nature of its capacity-building aim, attracts proposals from a range of research experience.

Lessons learnt: The TRGS is filling an important gap in the research funding landscape in NSW and is well regarded by stakeholders. To ensure that the TRGS is achieving its intended aims, an evaluation of the impact of the scheme will take place during 2018-19. 


\section{Introduction}

Bridging the 'gap' between research evidence and the complexities of policy and practice is an ongoing challenge. ${ }^{1}$ Ignoring this gap denies or delays the community's access to the most effective services and programs. ${ }^{2}$ Since 2015, the Translational Research Grants Scheme (TRGS) in New South Wales (NSW), Australia, has funded NSW Health services and specialty networks to conduct priority-driven clinical, health services and population health research. The NSW Ministry of Health (the Ministry) has the role of 'system manager' in relation to the NSW public health system, which operates public hospitals, as well as providing community health and other public health services, through a network of Local Health Districts, specialty networks and nongovernment affiliated health organisations, known collectively as NSW Health.

The TRGS is intended to drive practice change by embedding research within health services. The scheme has five main objectives:

- Foster the generation of high-quality research and evaluation that is directly relevant to clinical, health service and population health practice in NSW

- Support projects that have the potential to be translated into policy and practice

- Reduce the time from evidence generation to practice implementation

- Drive collaboration and practice change that improves health outcomes

- Improve health and medical research capability within the NSW health system.

The TRGS is unique in the Australian context as chief investigators must be employees of an approved NSW Health organisation: a NSW Local Health District (LHD); a Specialty Health Network (SHN); Ambulance Service of NSW; or NSW Health Pathology. This requirement places health service staff in a leadership role, unlike traditional research funding schemes which are generally led by researchers. ${ }^{3,4,5}$ Priorities for the scheme are drawn from state-wide health plans; local strategic plans; and strategies designed to support the translation of research into policy, practice and/or health services delivery. Applicants first submit an expression of interest (EOI) and can then be invited to submit a full application. There have been three TRGS funding rounds to date, and a fourth round opened in July 2018. Further information is available on the Office for Health and Medical Research website: www.health.nsw.gov.au/ohmr/Pages/trgs.aspx

\section{Translational Research Framework}

In order to foster a better understanding by the health service and research sectors about translational pathways, the NSW Ministry of Health collaborated with the independent organisation the Sax Institute to develop the TRGS Translational Research Framework (Figure 1). ${ }^{6}$ The framework describes a series of research steps moving from the development and testing of an innovative health service, program or policy; to testing the application of novel interventions that have worked in different circumstances or settings; through to systemwide application of innovations - reflecting the flow from innovation to system-wide testing and implementation at scale. Conceptual frameworks are important as they provide a common language to describe the processes and types of research associated with translation of research evidence into broader policy and practice. ${ }^{7}$ Applicants are encouraged to demonstrate an understanding of where proposed research projects sit on this continuum.

\section{Methods}

There were two stages to this review. Firstly an implementation review was conducted after round one of the TRGS. This involved a document review and semistructured interviews with key stakeholders involved in the first round. Data for the document review were extracted by two reviewers (RA and SR) and information was summated.

Interview participants included Ministry of Health staff involved in administering the TRGS and selection panel members $(n=8)$. Additionally, selected grant recipients $(n=4)$ across different LHDs and with differing research experience were interviewed to provide a breadth of views. The participants were asked about their opinions and experiences based on round one of the TRGS. Interviews were audio-recorded and subsequently transcribed by a third party with the participant's consent. Qualitative data were analysed through a thematic process by the two reviewers. Emerging themes were grouped according to relevant review questions.

Secondly, authors (TL and AP) have been involved in conducting quality improvement throughout the first three rounds of the scheme, through conversations with NSW Health staff involved in local coordination of the TRGS, and application reviewers.

\section{Results and discussion}

The first three TRGS funding rounds attracted 583 EOIs, with 53 projects receiving funding. NSW Health has committed $\$ 24,352,158$ across the three rounds to date. Due to the high demand in round one (348 EOIs), the process was changed to cap the number of EOls submitted. Round two received $172 \mathrm{EOIs}$; for round three, the cap was further reduced and 63 EOls were received. This encouraged the LHDs to conduct their own selection and prioritisation process. This also helped to make the TRGS more sustainable. Feedback to date indicates this process is useful because it also allows LHDs to plan 
Figure 1. Translational Research Framework: testing policy, program and service innovation

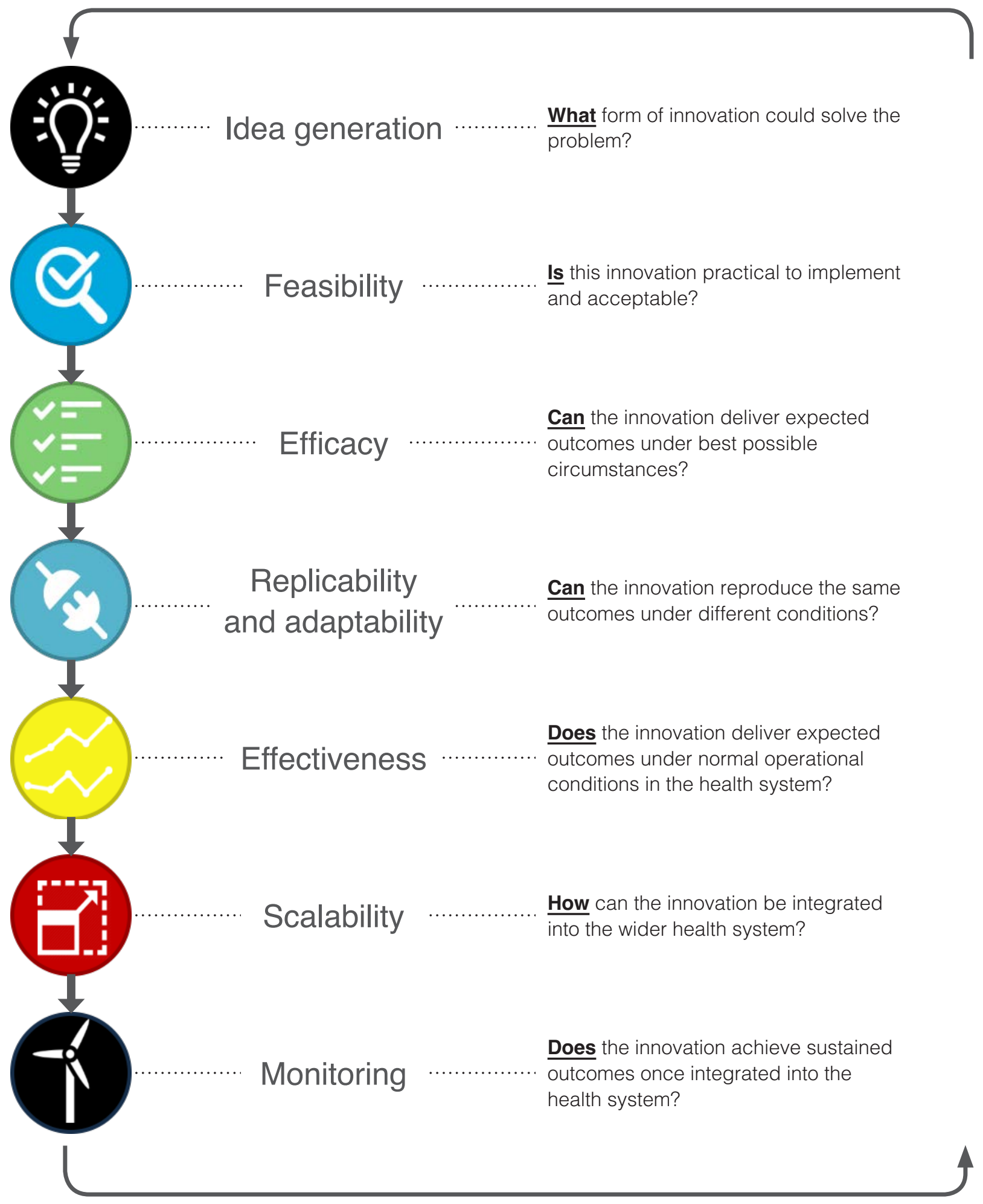

Source: Sax Institute ${ }^{6}$

a pipeline of research projects that can be supported locally or submitted to the TRGS. Since round two and the introduction of the local vetting process, some LHDs have established or improved their own research grants to support idea generation.

Since its inception, the TRGS has attracted a range of applicants from different LHDs, professions and levels of research expertise. Eighteen organisations across NSW Health have had projects funded. Of the funded projects led by an LHD $(n=48), 44 \%$ were led by a rural LHD. Over the three rounds, NSW Health and local priority research areas have been funded (Table 1). 
Table 1. Funded projects, by priority area

\begin{tabular}{lc}
\hline Priority area & $\begin{array}{c}\text { Number of } \\
\text { projects funded }\end{array}$ \\
\hline Aboriginal health & 2 \\
Cardiovascular disease & 7 \\
Childhood overweight and obesity & 8 \\
Diabetes & 4 \\
Drug and alcohol & 4 \\
Mental health & 9 \\
Other & 10 \\
Quality end-of-life care & 6 \\
Vulnerable young people and families & 3 \\
Total & $\mathbf{5 3}$ \\
\hline
\end{tabular}

\section{Implementation commitment}

Arguably, the most pivotal aspect of the scheme is the implementation commitment. Strategic visibility of the research projects from senior NSW Health staff during the review of EOls and full applications is supported by an implementation commitment at a local level. As part of the application process, Chief Executives of the host organisation (and some partner organisations) agree that practice change will be implemented if the results from the project provide evidence that practice change is merited. Two channels help to support implementation: visibility of project outcomes across the sector; and partnerships.

One [aim] was intentionally to get Chief Executive buy-in. Very important ... we wanted to have the Chief Executive support for this primarily because we want the findings of the research implemented and, as you know, that's one of the conditions of the application form. (TRGS selection panel member)

\section{Partnerships}

The host organisations are explicitly encouraged to establish partnerships with other health services, statewide agencies, clinical networks, peak bodies and relevant researchers before submitting applications. These policy and practice partnership requirements are intended to make funded research more relevant, generalisable and scalable across health systems.

Nearly all the projects that received funding in round one included multiple partners. Across the 24 projects, the average number of partners was five (range: 1-11). Round one-funded projects included more than 50 different partners in total, including university groups, peak bodies, other LHDs, medical research institutes, NSW Health Pillars and the NSW Ministry of Health.
Following round one, some recipients with less research experience found forging partnerships difficult:

And again that level of collaboration across different LHDs and agencies was quite difficult to achieve in the short time frame that I had. (TRGS recipient)

Building connections across the state and linking into state-wide priorities improved over the second and third rounds, possibly as a result of information sessions delivered by the Ministry.

\section{Focus on translational research}

Respondents believed the scheme was important in filling a funding gap for research in health services.

From a program overall perspective, I think actually having a research funding round which focuses on translation is really important. Most funding is either primarily targeted to the specific disease condition ... Alternatively, they tend to be more upstream, more experimental research grants, such as NHMRC [National Health and Medical Research Council] and ARC [Australian Research Council], and that's all very well and good but in healthcare, translation is nine-tenths of it. The experimental research is all well and good but there's a lack, in Australia, of opportunities for funding translation research into health systems and that's really important. That's pretty essential. (TRGS recipient)

\section{Capacity building}

Respondents identified that the TRGS: allows an opportunity to conduct research that was previously not easily undertaken by many health professionals; provides an opportunity for LHDs to advance their own research development initiatives; allows people employed by the LHD to be innovative about creating better care and services; and is building capacity by helping to establish and maintain important partnerships.

I think the other aspect of it is that it combines a research funding scheme with a building research capacity component, and I think it's very much promoting and supporting a partnership approach between the districts, breaking down barriers between districts and their local universities and MRIs [medical research institutes]. (Sub-committee member [involved in advising the EOI and selection panels])

Capacity building was also promoted via the process of providing feedback to applicants through the EOI and final application stages. The written and verbal feedback at full application stage helped applicants to strengthen their research proposal by enhancing rigour and alignment with priorities and partners. 
Some applicants found the feedback process helpful:

Definitely yes [the feedback was valuable in improving my project]. And l'd kind of gone from not $100 \%$ sure of what I was doing with the project to something that's really sound and really exciting, I feel really confident that we'll get good results out of it. And I think that's from the collaboration. The more people I've talked to, the more ideas have been able to develop and firm up. (TRGS recipient)

However, some applicants were critical of the feedback process, finding it inconsistent.

We believe feedback to applicants remains a valuable and essential part of the program delivery. While, at this stage, feedback is being provided only to those invited to submit a full application, consideration is being given to how to better provide constructive feedback to all applicants and the Chief Executives of the host organisations. The Ministry is also developing training resources for health system staff with varying levels of research design expertise.

Continued strategic oversight of the TRGS by the Chief Health Officer, Deputy Secretary Population and Public Health, and the Deputy Secretary Strategy and Resources ensures projects are strategically relevant to the health system.

Numerous resources have been added to the TRGS website to assist applicants, including checklists, generalised feedback from rounds one and two, contact details of Agency for Clinical Innovation Clinical networks, FAQs, and several videos from the Ministry information sessions. $^{8}$

\section{Conclusion}

The TRGS has been well received by those stakeholders interviewed, and has been implemented successfully in its first three rounds. The scheme has been successful in supporting health services-led research, and respondents suggest that it has also enhanced a culture of inquiry and innovation within the NSW health system. The requirement for links with clinical and policy networks at the outset and throughout the research project appears to have further embedded research within the NSW health system.

During 2018-19, an impact assessment of the TRGS will be conducted by an independent research group. The assessment will aim to capture the extent to which the findings from TRGS-funded projects are translated into policy and practice, and the extent to which the scheme has enhanced health and medical research capability. Measuring the effectiveness of the translation will be a key feature in further understanding the success of the TRGS.

\section{Peer review and provenance}

Externally peer reviewed, commissioned.

\section{Competing interests}

None declared.

\section{Author contributions}

RA, TL and AM were responsible for writing the paper, with direction, oversight and edits from AP and AM. RA and SR conducted most of the implementation review discussed throughout the paper.

\section{References}

1. Rychetnik L, Bauman A, Laws R, King L, Rissel C, Nutbeam D, et al. Translating research for evidencebased public health: key concepts and future directions. J Epidemiol Community Health. 2012;66(12):1187-92.

2. Milat AJ, King L, Bauman A, Redman S. The concept of scalability: increasing the scale and potential adoption of health promotion interventions into policy and practice. Health Promot Int. 2013;28(3):285-98.

3. National Health and Medical Research Council. Canberra: Commonwealth of Australia; 2018. NHMRC Project Grants; 2018 Jan [cited 2018 Feb 22]; [about 4 screens]. Available from: www.nhmrc.gov.au/grantsfunding/apply-funding/project-grants

4. National Institutes of Health: office of extramural research. Bethesda, MD: NIH. NIH Research Project Grant Program (R01); 2016 May [cited 2018 Feb 22]; [about 2 screens]. Available from: grants.nih.gov/grants/funding/r01.htm

5. Medical Research Council: leading science for better health. London: MRC; 2018. Research grant [cited 2018 Feb 22]; [about 3 screens]. Available from: www.mrc. ac.uk/funding/how-we-fund-research/research-grant/

6. Sax Institute. Translational Research Framework. Sydney: Sax Institute; 2016 [cited 2018 Feb 22]. Available from: www.health.nsw.gov.au/ohmr/Documents/trgs-round2translational-research-framework.pdf

7. Milat AJ, Li B. Narrative review of frameworks for translating research evidence into policy and practice. Public Health Res Pract. 2017;27(1):e2711704.

8. NSW Ministry of Health. Sydney: State of New South Wales (NSW Ministry of Health); 2018. Translational Research Grants Scheme; 2018 [cited 2018 Sep 6]; [about 2 screens]. Available from: www.medicalresearch. nsw.gov.au/translational-research-grants-scheme/

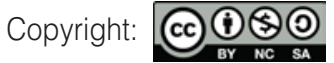

(c) 2018 Auld et al. This article is licensed under the Creative Commons Attribution-NonCommercial-ShareAlike 4.0 International Licence, which allows others to redistribute, adapt and share this work non-commercially provided they attribute the work and any adapted version of it is distributed under the same Creative Commons licence terms. See: www.creativecommons.org/licenses/by-nc-sa/4.0/ 